\title{
Caractérisation structurale des peuplements naturels de Khaya anthotheca (Welw.) C.DC dans les concessions forestières du Sud-Ouest de la République Centrafricaine
}

\author{
Bruce DEGUENE $^{1 *}$, Gorgon Igor TOUCKIA ${ }^{2}$, Olga Diane YONGO $^{2}$ et \\ Jean Joël LOUMETO ${ }^{3}$
}
${ }^{1}$ Institut Supérieur de Développement Rural (ISDR), Université de Bangui, BP : 1053, de M'Baïki, République Centrafricaine.
${ }^{2}$ Laboratoire de Biodiversité Végétale et Fongique, Faculté des Sciences, Université de Bangui, BP 908, Avenue des Martyrs, Bangui, République Centrafricaine.
${ }^{3}$ Laboratoire de Botanique et Ecologie, Faculté des Sciences et Techniques, Université Marien Ngouabi, BP : 69, Brazzaville, Congo.
*Auteur correspondant, E-mail : derembemi@yahoo.fr ; Tel : (+236) 75617077

\section{RESUME}

L'objectif de cette étude est d'apporter des informations scientifiques nécessaires à la préservation et gestion durable de Khaya anthotheca dans le sud-ouest de la République Centrafricaine via l'amélioration des connaissances sur son écologie. Pour y parvenir, des inventaires forestiers ont été effectués dans les Permis d'Exploitation et d'Aménagement (PEA) des entreprises forestières CentraBois (PEA 192) et Timberland (PEA188). Pour toutes les caractéristiques dendrométriques calculées en fonction des concessions et des classes de diamètre, le PEA 188 de Timberland est largement dominant. La densité moyenne des individus adultes est de 0,10 pieds/ha sur le PEA 192 et de 12 pieds/ha sur le PEA 188 avec le même coefficient de variation (cv) de $112,7 \%$. La surface terrière $\left(\mathrm{m}^{2} / \mathrm{ha}\right.$ ) varie de 0,86 sur le PEA 192 à 3,78 sur le PEA 188 avec des cv respectifs de $60,2 \%$ et $66,4 \%$. La densité de régénération moyenne varie de 3,52 plants/ha sur le PEA 192 à 12,19 plants/ha sur le PEA 188. Les courbes dendrométriques sont exponentielles décroissantes dans les deux concessions. Des travaux de recherches sur la régénération artificielle de $K$. anthotheca sont importantes pour définir des mesures de préservation dans ces zones.

(C) 2020 International Formulae Group. All rights reserved.

Mots clés : Concessions forestières, inventaires, régénération, gestion durable.

\section{Structural characterisation of natural populations of Khaya anthotheca (Welw.) C.DC in the forest concessions of South-West of Central African Republic}

\begin{abstract}
The objective of this study is to provide scientific information necessary for the preservation and sustainable management of Khaya anthotheca in the south-west of the Central African Republic by improving knowledge of its ecology. To achieve this, forest inventories were carried out in the Logging and Management Permits (PEA) of the forestry companies CentraBois (PEA 192) and Timberland (PEA188). For all dendrometric
\end{abstract}


characteristics calculated according to concessions and diameter classes, Timberland's PEA 188 is largely dominant. The average density of adult individuals is 0.10 tree/ha on PEA 192 and 12 tree/ha on PEA 188 with the same coefficient of variation $(\mathrm{CV})$ of $112.7 \%$. The basal area $\left(\mathrm{m}^{2} / \mathrm{ha}\right)$ varies from 0.86 on PEA 192 to 3.78 on PEA 188 with respective coefficient of variation of $60.2 \%$ and $66.4 \%$. The average regeneration density varies from 3.52 plant/ha on the PEA 192 to 12.19 plant/ha on the PEA 188. The dendrometric curves are exponentially decreasing in both concessions. Research on artificial regeneration of $K$. anthotheca is important to define preservation measures in these areas.

(C) 2020 International Formulae Group. All rights reserved.

Keywords: Forest concessions, inventories, regeneration, sustainable management.

\section{INTRODUCTION}

Khaya anthotheca (Welw.) C.DC., appelé encore Dèkè (en Issongo) en République Centrafricaine (RCA), est une espèce de forêts denses humides semi-sempervirentes rencontrée dans les deux blocs forestiers du Sud du pays et dans les galeries forestières. Elle est exploitée comme bois d'œuvre et comporte de nombreux autres usages (Maroyi, 2008; Touckia et al., 2014 ; Deguene et al., 2018 ; Bouka et al., 2019). Elle est largement utilisée par les populations rurales pour ses vertus médicinales, aphrodisiaques, mais également comme matériaux de construction, combustible, sculpture, etc. L'écorce, de saveur amère, est couramment utilisée en médecine traditionnelle et comme ingrédient dans le vin de palme. En décoction ou en infusion, elle se prend pour traiter la toux, la fièvre, les rhumes, la pneumonie, les douleurs abdominales, les vomissements et la gonorrhée ; en usage externe, elle s'applique sur les plaies, les écorchures et les ulcères. La poudre d'écorce se prend comme aphrodisiaque et pour traiter l'impuissance masculine (Hawthorne et al., 2006).

En République Démocratique du Congo (RDC), les feuilles sont utilisées pour fabriquer un poison de flèche (Maroyi, 2008). En Tanzanie, la décoction de racine se boit pour traiter l'anémie, la dysenterie et le prolapsus rectal. Dans ce même pays, l'écorce était jadis utilisée par la tribu des Shambaas pour teindre en brun rougeâtre. Le bois, du nom commercial acajou d'Afrique, African mahogany est tenu en haute estime pour la fabrication de meubles, l'ébénisterie, les boîtes, les casiers et les placages décoratifs. Il est aussi couramment employé pour les encadrements de fenêtres, les panneaux, les portes et les escaliers.
Traditionnellement, le bois est utilisé pour faire des pirogues monoxyles. Il est utilisé comme bois de feu et pour la production de charbon de bois (Ofori et al., 2007).

Ces nombreux usages cités ci-dessus, engendrent une pression sans cesse croissante sur le peuplement de $K$. anthotheca à l'état naturel à l'exemple d'autres espèces de plantes à usages multiples. Les études, sur certaines espèces telles que Afzelia africana (Sinsin et al., 2004), K. senegalensis (Gaoue et Ticktin, 2007) et de nombreuses plantes ligneuses d'Afrique centrale et occidentale (Traoré et al. 2011 ; Baye-Niwah et al., 2020) ont montré par exemple que l'exploitation des Produits Forestier Non Ligneux (PFNL) comme $K$. anthotheca peut affecter les paramètres vitaux, agissant sur la structure de leurs populations. Ces pressions anthropiques et autres facteurs de diverses natures agissent constamment sur la structure des populations de l'espèce sans que d'importantes actions spécifiques de conservation ne soient effectuées (Gbesso et al., 2014).

$K$. anthotheca, en particulier, figure sur la liste rouge de l'Union Internationale pour la Conservation de la nature (UICN) en tant qu'espèce vulnérable en raison de la perte et de la dégradation de son milieu, ainsi que de son abattage sélectif. Son inscription à l'annexe I ou II de la Convention sur le Commerce international des espèces de faune et flore sauvage menacées d'extinction (CITES) a été proposée, mais l'insuffisance d'informations relatives à sa régénération, à l'étendue des plantations et à sa durabilité avec les méthodes de gestion actuelles l'a écarté de cette liste (Hawthorne et al., 2006).

En RCA, il est noté une carence aigue des informations scientifiques sur la structure et 
la régénération des peuplements naturels de $K$. anthotheca en particulier et de nombreuses essences à usages multiples en général (RéjouMéchain et al., 2008; Béina, 2011). A cette carence, il faut aussi ajouter la difficulté liée à la confusion entre les espèces du genre Khaya souligné par Bouka et al. (2019) et qui contribuent à limiter les efforts de leur conservation et gestion durable. Pour pallier ce déficit, il s'est avéré important de conduire une étude pilote sur $K$. anthotheca, en particulier dans les concessions forestières du Sud-Ouest du pays où elle semble subir des pressions importantes et où elle a été classée comme « essence rare » dans le cadre de l'élaboration des plans d'aménagement de certaines concessions. L'amélioration des connaissances scientifiques attendue au terme de cette étude pourra permettre, par conséquent, de prévenir les menaces d'extinction, voir une proposition d'inscription de $K$. anthotheca à l'annexe 2 de la CITES.

C'est ce qui justifie cette étude dont l'objectif est d'apporter des informations scientifiques nécessaires à la préservation et la gestion durable des peuplements naturels de $K$. anthotheca dans les concessions forestières du sud-ouest de la RCA par l'amélioration des connaissances sur son écologie. De manière spécifique, il s'agit de : i) caractériser les peuplements naturels de $K$. anthotheca dans deux concessions forestières du sud-ouest de la RCA ; ii) évaluer le potentiel de régénération naturel de $K$. anthotheca dans ces deux concessions forestières.

\section{MATERIEL ET METHODES Sites d'étude \\ Localisation géographique}

La caractérisation de peuplements et l'étude de régénération de $K$. anthotheca ont été conduites de manière comparative dans deux concessions forestières du Sud-ouest de la RCA.

Le lot $\mathrm{A}$ de la concession forestière de l'entreprise CentraBois (PEA 192), situé dans le bloc Est du massif forestier du Sud-ouest de la RCA, est le premier site de la présente étude. Cette concession est située dans les préfectures de l'Ombella-M'Poko (la grande partie) et de la Lobaye (pour une petite portion) dans le Sud-
Ouest de la RCA. Elle s'étend plus précisément entre $3^{\circ} 34^{\prime}$ ' et $2^{\circ} 36^{\prime}$ de latitude Nord et ; $16^{\circ} 10^{\prime}$ et $16^{\circ} 40^{\prime}$ de longitude Est, couvrant ainsi, une superficie de plus de 156817 ha.

Cette concession forestière a subi plusieurs passages de l'exploitation industrielle d'une part, et aussi la pression de l'agriculture itinérante ainsi que d'autres usages des populations d'autre part. Elle est ainsi composée de juxtaposition de surfaces agricoles, des jachères composant les terroirs villageois et des zones de forêts dégradées ou denses, et aussi de savanes (Figure 1).

D'une superficie de 229025 ha, la concession forestière de Timberland (PEA 188) située dans le «Bloc Ouest» du massif forestier du Sud-ouest de la RCA est le second site de cette étude. L'essentiel du PEA 188 est situé dans la préfecture de la Sangha-Mbaéré, une petite partie est située dans la préfecture de la Mambéré-Kadéí (Figure 2). Elle s'étend précisément entre $3^{\circ} 20^{\prime}$ et $4^{\circ} 05^{\prime}$ de latitude Nord et $15^{\circ} 35^{\prime}$ et $16^{\circ} 10^{\prime}$ de longitude Est. Elle se trouve dans le faciès typique de forêt semicaducifoliée dont le groupe d'essences caractéristiques comprend les espèces suivantes; Celtis zenkeri (Ohia parallèle), Triplochiton scleroxylon (Ayous), Duboscia macrocarpa (Kakama), Terminalia superba (Fraké), Anonidium mannii (Mobaï), etc.

\section{Climat}

Le climat régnant sur les deux concessions forestières est du type guinéen forestier avec une pluviométrie caractérisée par l'alternance d'une courte saison sèche de novembre à février et une saison des pluies le reste de l'année. Le total des précipitations s'élève à 1600 mm par an (MEFCP, 2018).

\section{Température et humidité de l'air}

Les températures moyennes annuelles varient entre $24{ }^{\circ} \mathrm{C}$ et $26{ }^{\circ} \mathrm{C}$ et l'humidité relative reste élevée au cours de l'année (80\%) sur le PEA 188. Sur le PEA 192, les températures annuelles demeurent constamment élevées, avec une moyenne annuelle de $26{ }^{\circ} \mathrm{C}$ relevés sur Bangui, à proximité du PEA. Les températures les plus basses sont observées entre les mois de décembre et janvier alors que les températures les plus élevées sont observées en mars sur cette dernière concession forestière (MEFCP, 2018). 
Sols

La plus grande proportion des sols des deux concessions forestières est principalement de type « ferrallitique ». Des sols à minéraux bruts et les sols hydromorphes sont aussi rencontrés sur le PEA 188 (MEFCP, 2018).

\section{Hydrographie}

Le réseau hydrographique du PEA 188 est composé principalement de deux grands cours d'eaux qui, avec leurs affluents, drainent la zone du PEA. Il s'agit de la Kadéï et de la Mambéré. Par contre, les principales rivières que l'on rencontre sur le lot A du PEA 192 sont l'Oubangui, la Pama et la Lessé (MEFCP, 2018).

\section{Végétation}

Le massif forestier du Sud-Ouest de la RCA, est situé dans le domaine congo-guinéen. C'est une forêt semi-décidue à Ulmacées, Sterculiacées, Sapotacées et Méliacées. Des arbres géants comme Autranella congolensis (Mukulungu), Manilkara letouzei (Manilkara) et Entandrophragma seraient les témoins d'une ancienne forêt primaire. Ricinodendron heudelotii (Essessang), Pycnanthus angolensis (Ilomba) et Triplochiton scleroxylon (Ayous) traduirait une secondarisation plus ou moins ancienne (MEFCP, 2018).

\section{Population}

Le nombre total d'habitants sur le PEA 192 est de 39667 soit une densité de 23,4 habitants au $\mathrm{km}^{2}$, largement supérieur à la moyenne nationale, qui est de 6,3 habitants au $\mathrm{km}^{2}$ (MEFCP, 2018). Sur le PEA 189 de Timberland, la population est estimée par contre à 63000 habitants soit une densité de 3,6 habitants au $\mathrm{km}^{2}$ (MEFCP, 2019).

\section{Approche méthodologique}

\section{Inventaire forestier de Khaya anthotheca (Welw.) C.DC.}

Les données d'inventaire des individus de $K$. anthotheca utilisées dans le cadre de cette étude, ont été collectées lors des inventaires d'aménagement des concessions forestières des entreprises Centrabois et Timberland (Figures 1 et 2 respectivement), réalisés sur la période de 2017 à 2018. Ces données sont disponibles dans les rapports d'inventaire de ces deux concessions forestières.
Les sondages sur ces deux concessions ont été réalisés par échantillonnage systématique, avec des placettes contiguës positionnées sur des layons équidistants et parallèles ouverts en forêt, souvent perpendiculaires à l'orientation générale du réseau hydrographique. Le but était de permettre à ce que l'échantillonnage représente au mieux la variabilité des formations végétales (Doucet, 2003).

Des layons de comptage sont positionnés à intervalles réguliers de $1600 \mathrm{~m}$, perpendiculairement au layon de base et orientés Est-Ouest. Quelques layons orientés dans le sens Nord-Sud ont également été prévus pour compléter le dispositif. Ceux-ci sont positionnés à intervalles réguliers, perpendiculairement aux layons de base. La placette, unité élémentaire d'échantillonnage, est assise longitudinalement sur le layon de comptage. Elle a une surface de 0,5 ha avec 200 $\mathrm{m}$ de long et $25 \mathrm{~m}$ de large (soit $12,5 \mathrm{~m}$ de chaque côté de l'axe central). Au total, le dénombrement a été effectué dans 105 placettes dans le PEA 192 de Centrabois et 141 placettes dans le PEA 188 de Timberland.

Dans chaque placette, le diamètre à 1,30 $\mathrm{m}$ du sol (DBH) de tous les pieds de $K$. anthotheca est mesuré. Cette mesure concerne les individus ayant plus de $10 \mathrm{~cm}$ de diamètre. Les pieds de DBH inférieur à $10 \mathrm{~cm}$ sont pris en compte dans l'étude de la régénération potentielle de cette espèce.

Les traces d'exploitation éventuelle (coupe complète, collecte des fruits) de chaque pied ont été notées. Les différentes pressions qui s'exercent sur les populations de l'espèce étudiée ont également été identifiées.

\section{Collecte des données pour la régénération naturelle de $\mathrm{K}$. anthotheca}

Lors des sorties sur le terrain, des pieds de semenciers de $K$. anthotheca ont été identifiés dans les séries agricoles des deux concessions cibles de l'étude. Au pied de chaque individu identifié, huit (08) couloirs (ou transects) de $50 \mathrm{~m} \times 1 \mathrm{~m}$ (soit $8 \times 50 \mathrm{~m}^{2}$ ) (Kasaï, 2007), ont été tracés dans les directions E-O, N$\mathrm{S}$, NE-SO et NO-ES avec une boussole et à l'intérieur desquels, les plantules de $K$. anthotheca sont comptées dans des microparcelles (ou placeaux) de $5 \mathrm{~m} \mathrm{x} 1 \mathrm{~m}$ (soit $5 \mathrm{~m}^{2}$ ). 
Le choix des semenciers dans les séries agricoles est guidé par la présence des plantules sous les arbres et la méthodologie appliquée est celle de Dansereau et Lems (1957) telle qu'utilisée par Doucet (2003).

\section{Traitement et analyse statistique des données}

Pour déterminer les caractéristiques dendrométriques de $K$. anthotheca, des paramètres dendrométriques ont été calculés par concession forestière (PEA 192 et PEA 188) afin de comparer les différents peuplements. Il s'agit notamment de la densité, la surface terrière et le volume moyen. Ces paramètres sont repris dans le Tableau 1.

La surface terrière totale de $K$. anthotheca est considérée comme la somme des sections de tous les individus de cette espèce qui constituent un peuplement si on les tronçonnait à $1,30 \mathrm{~m}$ du sol. Elle s'exprime en mètre carrés par hectare $\left(\mathrm{m}^{2} / \mathrm{ha}\right)$.

Les caractéristiques structurales (densité, distribution des individus par classes de diamètre et de volume) ont été calculées en déterminant les moyennes par concession forestière. Pour chaque valeur obtenue, le coefficient de variation est calculé.

Les arbres ont été regroupés en 8 classes de diamètre d'amplitude $10 \mathrm{~cm}$ comme suit :

- Classe 1 = DBH situé entre10 et 19,99 cm

- Classe $2=\mathrm{DBH}$ allant de 20 à $29,99 \mathrm{~cm}$

- Classe $3=$ DBH30 à 39,99 cm,

- Classe 4 = DBH40 à 49,99 cm,
- Classe $5=$ DBH50 à 59,99 cm,

- Classe $6=$ DBH60 à $69,99 \mathrm{~cm}$,

- Classe $7=$ DBH70 à 79,99 cm

- Classe $8=\mathrm{DBH}=80 \mathrm{~cm}$.

La dernière classe correspond au Diamètre Minimum d'Aménagement (DMA) du $K$. anthotheca à $80 \mathrm{~cm}$ tel que fixé dans les documents portant respectivement convention définitive et convention provisoire d'exploitation et d'aménagement des deux concessions forestières de CentraBois et Timberland, cibles de la présente étude (MEFCP, 2019 et 2018).

Une analyse de variance (ANOVA) à deux facteurs avec le logiciel R 3 6.2, a été effectuée sur les matrices de données contenant les variables densité, surface terrière, volume et nombre de plants régénérés par pied de semencier de $K$. anthotheca, par classe de diamètre et par concession forestière.

La densité des plants issus de la régénération naturelle a été aussi calculée pour évaluer et apprécier l'état de régénération de $K$. anthotheca dans son habitat et en fonction des deux PEA (188 et 192). Ces données ont été également soumises à une analyse de variance dans $\mathrm{R}$, en vue de comparer la densité de régénération en fonction des classes de diamètre des semenciers, des concessions et des différentes orientations géographiques (directions préférentielles de régénération).

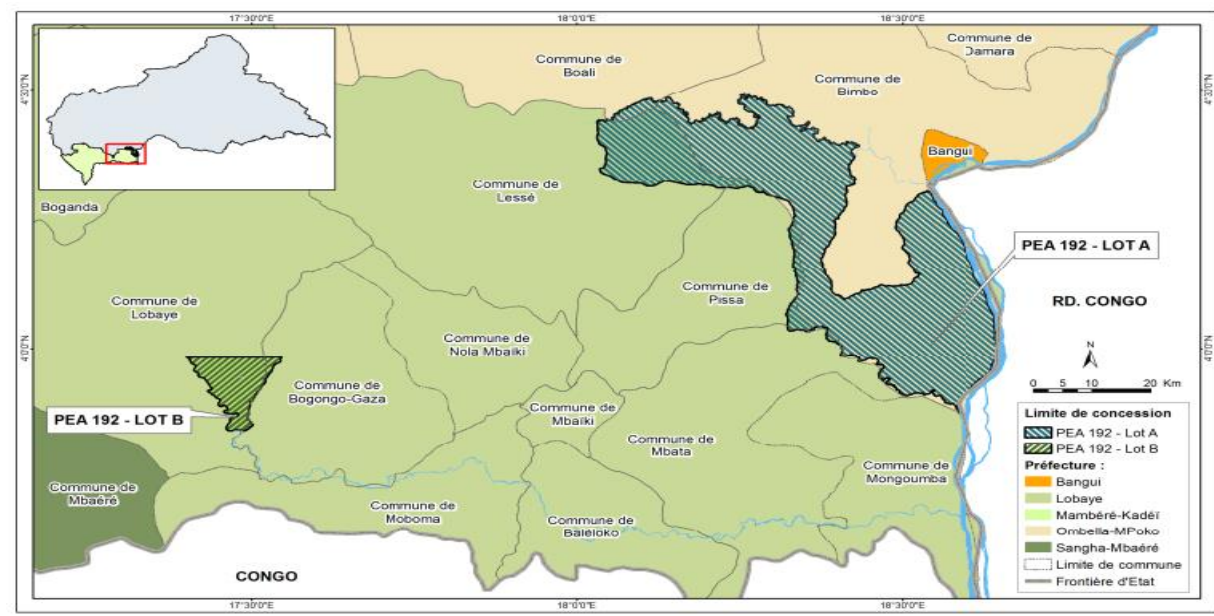

Figure 1 : Localisation de la concession forestière de l'entreprise CentraBois (MEFCP, 2018). 


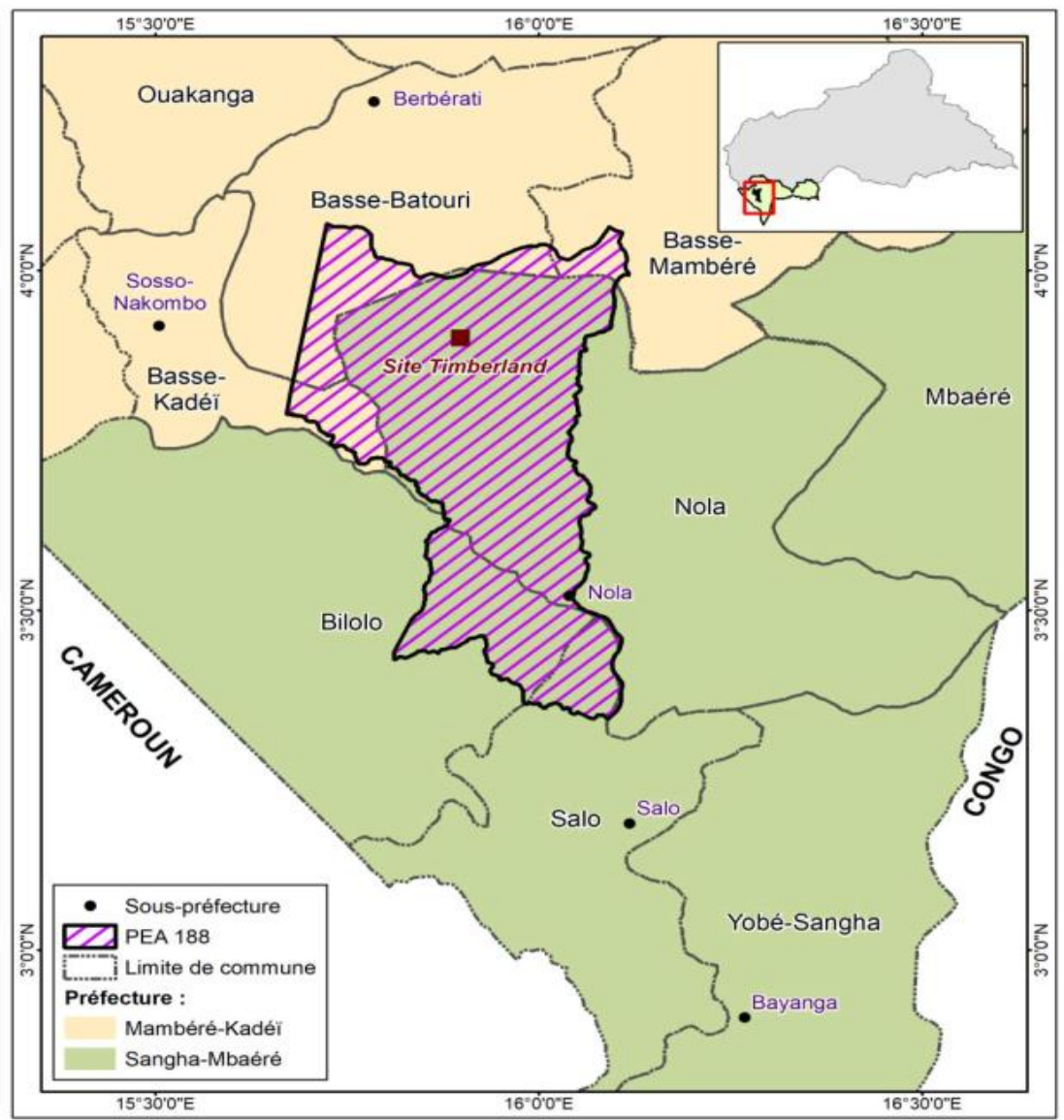

Figure 2 : Localisation de la concession forestière de l'entreprise Timberland (MEFCP, 2019).

Tableau 1 : Paramètres dendrométriques et leurs formules.

\begin{tabular}{|l|l|}
\hline Paramètres & Formules \\
\hline Densité $($ pieds/ha) & $\mathrm{N}=$ Nombre total de pieds/superficie \\
\hline Surface terrière $\left(\mathrm{m}^{2} / \mathrm{ha}\right)$ & $\mathrm{G}=\sum \pi \mathrm{D}^{2} / 4$ \\
\hline & $\mathrm{V}=\sqrt{\frac{1}{\mathrm{n}} \sum_{\mathrm{i}=1}^{\mathrm{n}} \mathrm{V}_{\mathrm{i}}^{2}}$ \\
\hline & \\
\hline
\end{tabular}

\section{RESULTATS}

\section{Caractéristiques dendrométriques}

Pour toutes les caractéristiques dendrométriques (densité, surface terrière, volume, densité des plantules) du K. anthotheca étudiées, la concession forestière de Timberland (PEA 188) est largement dominant
(Tableau 2). On peut noter au regard des chiffres que le nombre des individus décroît du PEA 188 (situé plus au Sud-Ouest) vers le PEA 192 situé dans l'extrême Est de ce même massif forestier. La surface terrière moyenne est de $0,02 \mathrm{~m}^{2} / \mathrm{ha}$ dans le PEA 192 et de $3,78 \mathrm{~m}^{2} / \mathrm{ha}$ dans le PEA 188 avec des coefficients de 
variation oscillant faiblement de $60,2 \%$ sur le PEA 192 à 66,4\% sur le PEA 188. Au niveau des volumes moyens à l'hectare, il est de 0,19 $\mathrm{m}^{3} /$ ha sur le PEA 192 tandis qu'il culmine à $45 \mathrm{~m}^{3} /$ ha sur le PEA 188 . Avec des coefficients de variation oscillant entre $31 \%$ sur le PEA 192 à $62 \%$ sur le PEA 188, on note une grande variabilité dans la distribution de ce paramètre au niveau des deux concessions forestières cibles de l'étude. Enfin, au niveau de la régénération naturelle, les résultats de l'étude montrent aussi une grande différence entre les deux sites de l'étude avec une moyenne de 12,19 plantules par semencier sur le PEA 188 tandis que cette valeur décroit jusqu'à 3,52 sur le PEA 192. L'analyse de la variance montre une différence hautement significative ( $\mathrm{P}$ value $=0,001)$.

\section{Structure démographique de Khaya anthotheca}

La structure démographique de $K$. anthotheca présente une allure exponentielle décroissante des petits diamètres vers les gros diamètres dans les concessions forestières de Timberland PEA 188) et de CentraBois (PEA 192) de façon générale (Figure 4). La comparaison de la densité des individus à l'hectare, montre cependant que le $K$. anthotheca est plus présent sur le PEA 188 à l'inverse du PEA 192. En effet, sur le PEA 188, le nombre des individus varie entre 1 à 5 pour le petits diamètres $(\mathrm{D}<=30 \mathrm{~cm})$ tandis que l'on retrouve moins d'un individu à l'hectare sur le PEA 192 pour toutes les différentes classes de diamètre. Les individus ayant atteint le DMA $(80 \mathrm{~cm})$ sont trop peu nombreux dans les deux concessions forestières cibles de l'étude avec moins d'un individu par hectare. Ce qui justifierait l'interdiction d'exploitation dans le PEA 192.

\section{Surface terrière de $K$. anthotheca par classe de diamètre dans les deux PEA}

Les résultats de l'étude montrent de manière générale une diminution de la surface terrière des petits diamètres vers les plus gros diamètres dans les deux concessions forestières cibles (Figure 5). Ce qui se justifie par le nombre réduit des individus de gros diamètre. De même, comme pour la structure diamètrique, les surfaces terrières présentent des valeurs plus importantes sur le PEA 188 (oscillant vers 1 pour les petits diamètres) tandis que celles-ci sont réduites sur le PEA 192. Ceci est en lien avec la rareté des individus de cette espèce dans cette concession. D'un point de vue statistique, la différence observée entre les deux PEA est significative $(\mathrm{p}$-value $=$ 0.01354).

\section{Volume mobilisable de $K$. anthotheca par classe de diamètre}

Le volume qui exprime la disponibilité de la ressource mobilisable dans le cadre d'une possible opération d'exploitation, varie aussi des petits diamètres vers les gros diamètres dans les deux sites de cette étude. En tenant compte du DMA de $K$. anthotheca, les résultats montrent cependant que le PEA 188 dispose plus de volume de bois mobilisable par rapport au PEA 192 avec une différence observée hautement significative $(\mathrm{p}$-value $=0.00807)$ entre les deux.

\section{Régénération naturelle de $K$. anthotheca Régénération de K. anthotheca en fonction de Classe de diamètre et de concession}

La Figure 7 présente le nombre de plants de $K$. anthotheca régénérés en fonction de classes de diamètre, dans les deux concessions forestières. Les plantules sont notées à partir de la classe de diamètre 4 . Leur nombre est plus élevée dans le PEA 192, pour les classes de diamètre 4,5 et 6 . Par contre, pour la classe 7 le nombre de plantules régénérées est similaire dans les deux concessions forestières.

Les résultats de l'analyse de variance montrent de manière globale que la classe de diamètre influence la régénération de façon significative $(\mathrm{P}$-value $=0,018)$. De même, la régénération varie en fonction des concessions forestières avec une différence observée hautement significative $(\mathrm{P}=2,2 \mathrm{e}-16)$. Enfin, l'interaction entre les classes de diamètre et les concessions forestières influence aussi la régénération de cette espèce avec une 
différence observée très significative $(\mathrm{P}=0,0075)$.

\section{Direction préférentielle de régénération de Khaya anthotheca}

La Figure 8 illustre la répartition des densités de semis de $K$. anthotheca suivant huit orientations géographiques. Les résultats obtenus montrent que sur le PEA 192, la valeur de densité la plus élevé est dans la direction Sud-ouest (22\%), tandis que la valeur plus faible est dans la direction Ouest (4\%). Sur le PEA 188 par contre, la valeur la plus forte est obtenue à l'Est (21\%), et la plus faible au Sud (4\%). Les résultats de l'analyse de variance de la densité de régénération en fonction des huit (8) directions dans les deux concessions confirment cette tendance avec une différence hautement significative $(\mathrm{P}$-value $=0,00064)$ observée.

En conséquence des observations cidessus, il n'y a pas une direction préférentielle de régénération de $K$. anthotheca car les valeurs varient d'une concession forestière à l'autre.

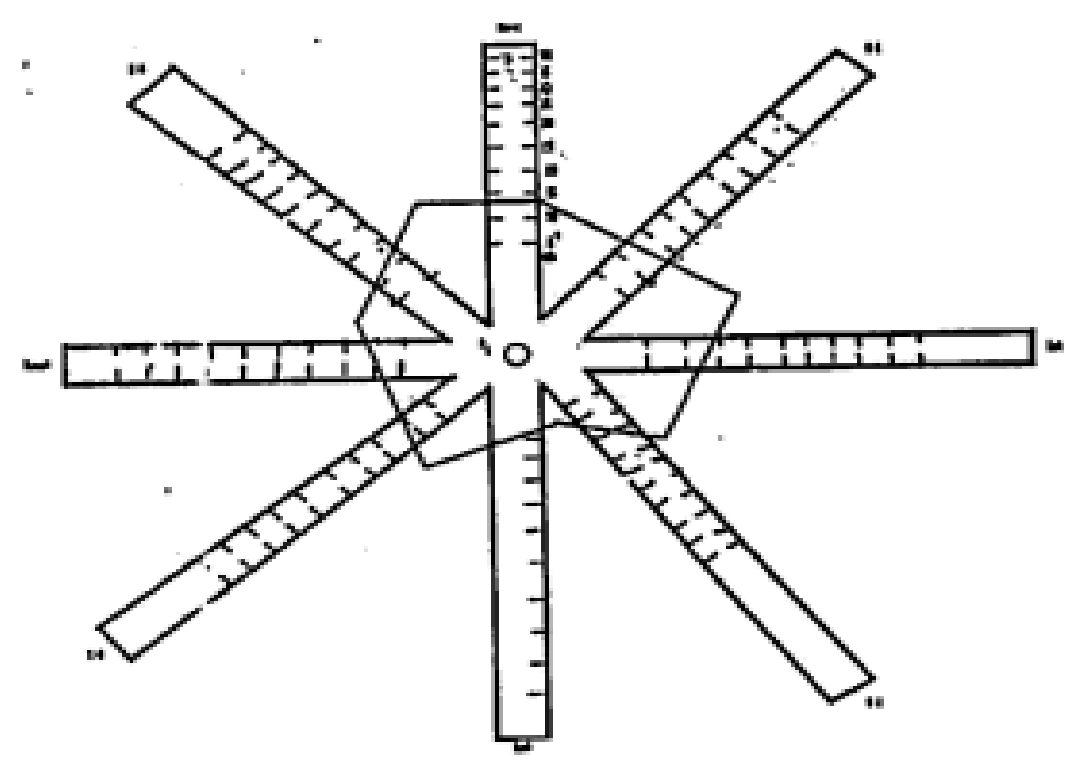

Figure 3 : Dispositif de collecte des données de régénération dans les couloirs (ou transects).

Tableau 2: Caractéristiques dendrométriques des individus de $K$. anthotheca dans les deux concessions forestières étudiées du Sud-ouest de la RCA.

\begin{tabular}{|c|c|c|c|c|}
\hline \multicolumn{5}{|c|}{ Individus adultes } \\
\hline \multirow{2}{*}{ Paramètres } & \multicolumn{2}{|c|}{ PEA 192} & \multicolumn{2}{|c|}{ PEA 188} \\
\hline & Moyenne & $\mathrm{CV}(\%)$ & Moyenne & $\mathrm{CV}$ \\
\hline N (pieds/ha) & 0,10 & 112,7 & 12,00 & 112,7 \\
\hline $\mathbf{G}\left(\mathbf{m}^{2} / \mathrm{ha}\right)$ & 0,86 & 60,2 & 3,78 & 66,4 \\
\hline $\mathbf{V}\left(\mathbf{m}^{3}\right)$ & 0,19 & 31,0 & 45,40 & 62,0 \\
\hline \multicolumn{5}{|c|}{ Régénération naturelle } \\
\hline $\mathbf{N}$ (plants/ha) & 3,52 & 87,5 & 12,19 & 58,1 \\
\hline
\end{tabular}




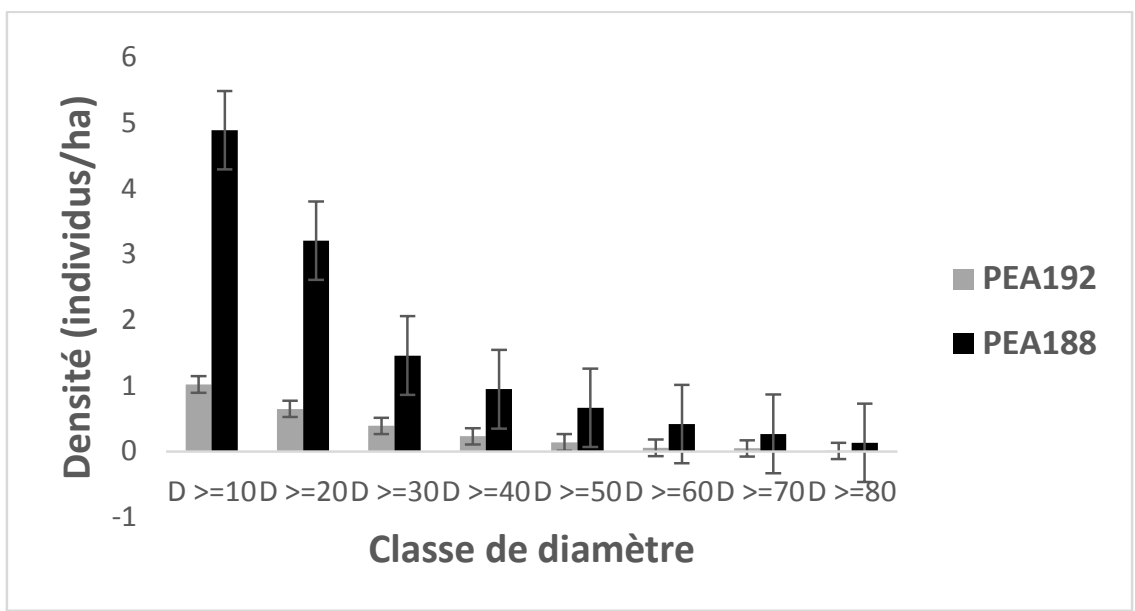

Figure 4 : Répartition de $K$. anthotheca par classes de diamètre sur le PEA 192 et le PEA 188.

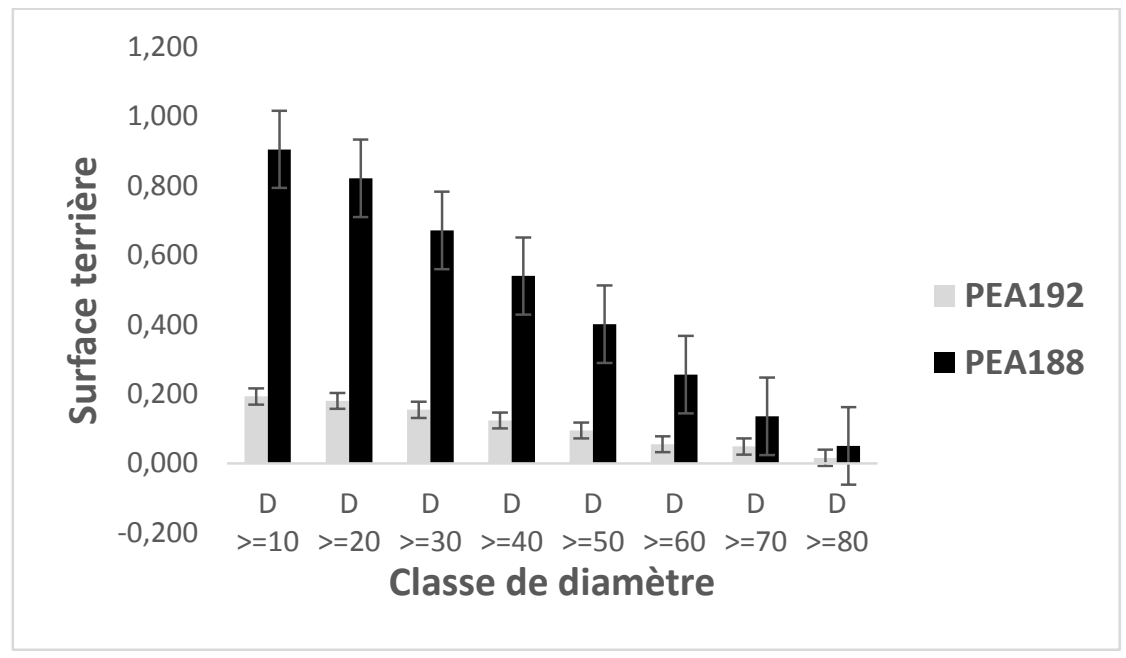

Figure 5 : Surfaces terrières de K. anthotheca par classes de diamètre sur les PEA 192 et le PEA 188.

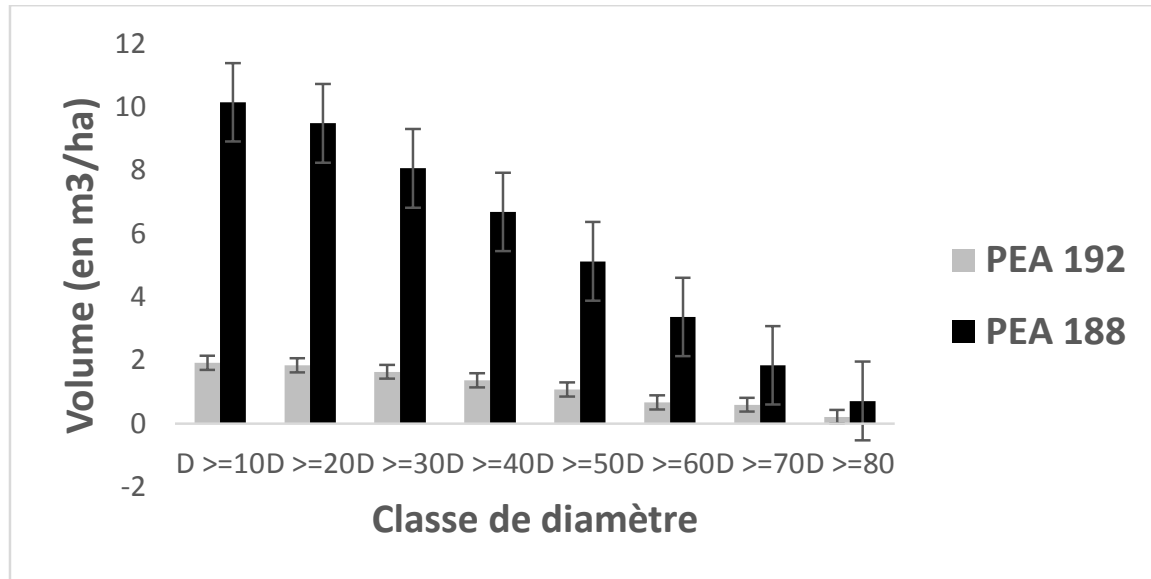

Figure 6 : Volume de K. anthotheca par classe de diamètre sur le PEA 192 et le PEA 188. 


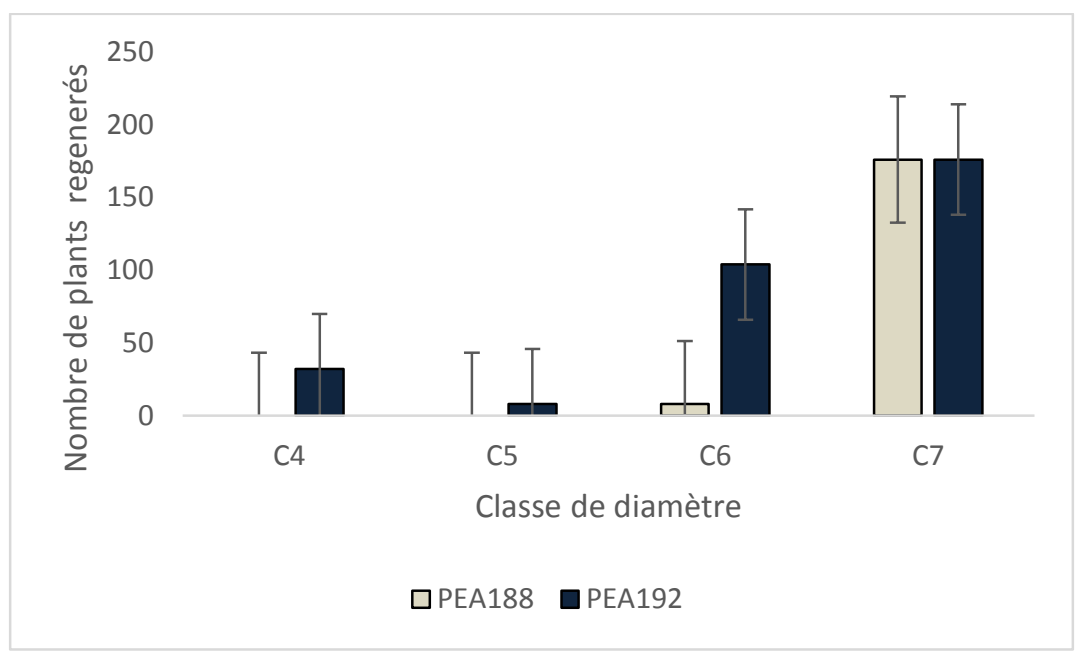

Figure 7 : Nombre de plants de $K$. anthotheca régénérés par classes de diamètres et par concession forestière.

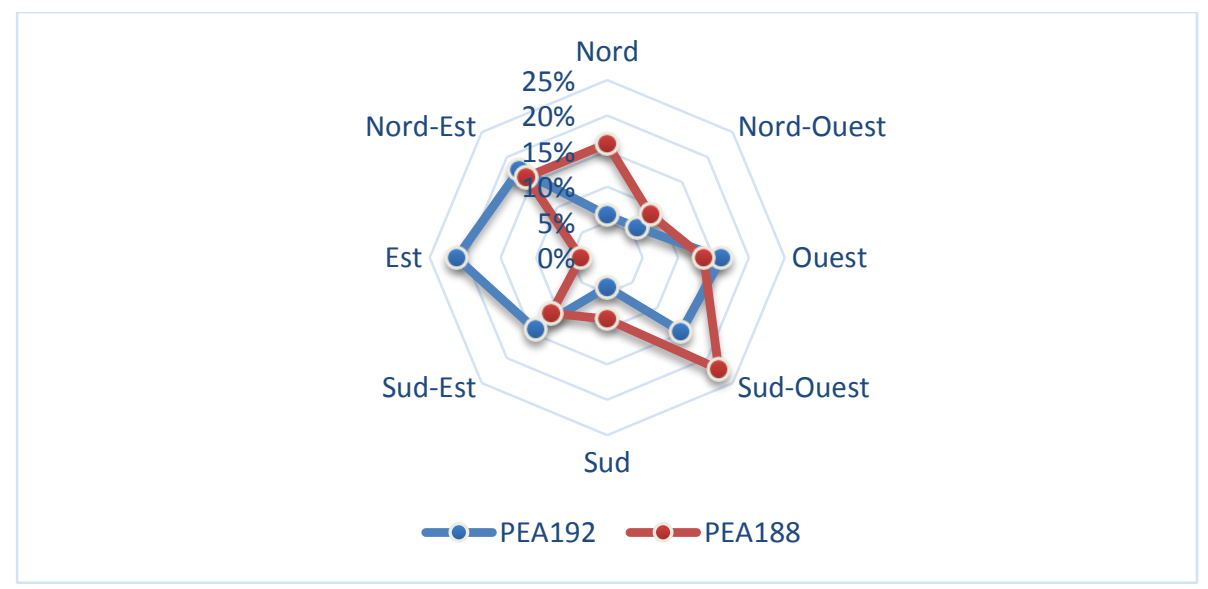

Figure 8 : Régénération de $K$. anthotheca en fonction des directions géographiques.

\section{DISCUSSION}

\section{Influences des paramètres dendrométriques} sur la régénération naturelle de $K$. anthotheca

Les faibles densités de $K$. anthotheca dans les deux concessions forestières peuvent s'expliquer par plusieurs raisons. La première, avancée dans le cadre de l'aménagement forestier en RCA, serait que cette espèce se situerait à la limite ou en dehors de son aire de répartition géographique (MEFCP, 2018).

En second lieu, cette faible densité de $K$. anthotheca dans les deux concessions pourrait s'expliquer principalement par les pressions humaines liées à ses diverses utilisations ethnobotaniques et les prélèvements répétés dans le cadre de l'exploitation industrielle passée. Ces pressions vont croissant du PEA 188 situé plus à l'ouest vers le PEA 192 situé dans les zones d'influence de la capitale Bangui. On peut noter comme pressions humaines, l'écorçage, le prélèvement des racines et des feuilles pour des usages ethnobotaniques divers (Traoré et al, 2020, Deguene et al., 2018 ; Hawthorne et Jongkind, 2006). A ces usages, il faut ajouter les dégâts de l'exploitation forestière sur les peuplements résiduels, les défrichements pour la mise en place des parcelles agricoles, etc. (Deguene et al, 2018; Maroyi, 2008). Les 
travaux de Traoré et al (2020), Sourou (2017), Houéssou et al. (2012) et Orékan (2007) ont fait cas des pressions telles que les feux de végétation et les différentes activités humaines qui contribuent à la réduction de la densité des espèces dans leurs différents habitats. Dans les séries agricoles et de développement humain $(\mathrm{SAOH})$ de ces deux concessions forestières, il est noté une quasi absence des individus adultes, ce qui témoigne de l'impact négatif de la pression humaine sur la densité des espèces. De tels résultats mentionnant la faible densité des espèces dans les agrosystèmes ont été obtenus par Gouwakinnou et al. (2009).

La densité de régénération de $K$. anthotheca est aussi très faible sur le PEA 192 $(3,52)$ et relativement élevé sur le PEA 188 (moyenne de 12,19 plantules par pied de semencier). Kasaï (2007) dans une étude similaire sur la régénération de $K$. anthotheca dans la réserve forestière de Yoko en RDC avait en effet obtenu une valeur moyenne de huit (8) plantules par pied de semencier. Ceci démontre la grande difficulté pour cette espèce d'assurer sa survie sur le PEA 192, et qui peut à terme contribuer à sa disparition dans les zones forestières situées proches des grandes agglomérations à l'exemple de la capitale Bangui. De même que pour les pieds adultes, la faible densité des plantules de $K$. anthotheca pourrait s'expliquer par les pressions humaines ainsi que les phénomènes naturels liés à chacun des habitats de cette espèce. Comme pressions humaines, on peut citer les piétinements des plantules lors de l'écorçage des adultes, l'arrachage des jeunes plantules pour des usages médicinaux, etc. (Deguene et al, 2018). Comme phénomènes naturels, on pourra citer l'absence de contact des graines avec le sol et $\mathrm{du}$ non enfouissement d'où l'absence de germination (Makana et Thomas, 2004). Les graines de $K$. anthotheca tombent et restent audessus des feuilles mortes et finissent par pourrir. Dans le PEA 192, de nombreux pieds de semencier ont été identifiés en bordure des cours d'eau. Ceci suppose, que les graines, en tombant, germent même lorsqu'elles se trouvent sur les feuilles mortes (en profitant de l'humidité des bordures de cours d'eau). Mais, au moment des crues, les jeunes plantules sont emportées par le courant vers l'aval compromettant ainsi leur survie et posant ainsi un véritable problème pour la régénération de cette espèce dans son habitat. La plupart des pieds de $K$. anthotheca retrouvés au bord des cours d'eaux sont inclinés dans le lit des cours d'eaux. Pendant la période de fructification une grande partie des graines mûr tombent dans les points d'eaux dans le lit des cours d'eaux ce qui constitue aussi un facteur qui ralentit le taux de germination de ces graines. Tout ceci explique donc de la variabilité de la densité des régénérations naturelles en fonction des différentes formations végétales. $\mathrm{Ce}$ qui exprime l'impact négatif de la formation végétale sur la densité et survie des régénérations des espèces. Les travaux de Sourou et al. (2016), Bamba et al. (2008), repris par Dossa et al. (2020) ont fait cas de l'impact de la géomorphologie sur la faible densité des régénérations. Il a par ailleurs souligné dans le cas de l'étude sur Detarium senegalense, que la conservation des espèces et de leurs capacités à s'adapter aux dérèglements dans leurs différents habitats constituent une représentativité des individus de ces espèces dans les différentes classes d'âges (Bamba et al., 2008). Ceci révèle donc la vulnérabilité et l'extinction des individus des espèces ayant de faible densité comme le cas de $K$. anthotheca. La faible densité de ces espèces est représentée par leurs classes d'âges largement inférieures lorsqu'elles sont soumises à l'exploitation humaine ou à d'autres formes de perturbations internes ou externes à leurs habitats (Kourouma et al, 2013).

\section{Impact des pressions anthropiques sur la structure démographique de Khaya anthotheca}

Deux grands types de distributions diamétriques sont couramment distingués : les distributions de type exponentielle décroissante pour les essences sciaphiles et les distributions en « cloche » pour les essences héliophiles (Doucet, 2003). Dans le cadre de la présente étude, les courbes de distributions diamétriques présentent la même allure au niveau des deux 
concessions forestières. Elles sont toutes deux de type exponentielle décroissante des plus petits diamètres vers les gros diamètres. $K$. anthotheca appartient donc au groupe des sciaphiles au stade jeune. Cependant, la prépondérance des individus de petits diamètres ne signifie pas que cette espèce dispose d'une niche de régénération abondante car il est souligné plus haut qu'il y a un déficit qui peut compromettre à terme la survie de l'espèce dans la zone forestière du Sud-Ouest. Il y a la persistance de risques naturels de mortalité en lien avec cette dominance des plantules comme souligné par Ahissou et al. (2017) dans le cadre de l'étude sur la caractérisation structurale des populations de Borassus aethiopum dans la commune de Savè au Bénin. Les individus de gros diamètre du fait des différents usages ethnobotaniques et de l'exploitation passée, sont soumis à une grande pression compromettant la viabilité de la population de l'espèce, ce qui confirme donc sa vulnérabilité (Neuenschwander et al., 2011). Ce risque est encore plus grand lorsqu'on constate que la densité des individus de grand diamètre est très faible de l'ordre de 0,01 individu à l'hectare sur les deux concessions forestières. La destruction des jeunes plants lors des prélèvements des écorces et racines des individus adultes ne favorise pas la survie des premières ce qui contribue de même à rendre plus vulnérable cette espèce. La mise en place des mesures d'assistance à la régénération naturelle (RNA) pourrait contribuer à garder cette allure et assurer la survie à moyen et long terme de $K$. anthotheca dans la région forestière du Sud-Ouest de la RCA. A contrario, on pourra, à terme, assister à une inversion de cette courbe vers une allure en cloche caractéristique des espèces dont le peuplement subit des perturbations profondes (Dossa, 2019). Les individus de plus gros diamètres sont relativement mieux représentés sur le PEA 188. Cela met en évidence une fois de plus l'impact des pressions sur le peuplement dans le PEA 192 qui est plus proche de Bangui à l'inverse du PEA 188 dont l'éloignement, contribue à une relative préservation des individus de gros diamètre. En terme de volume mobilisable dans le cadre d'une exploitation forestière, l'étude a montré que le PEA 188 est mieux fourni en comparaison du PEA 192.

\section{Densité et direction préférentielle de régénération naturelle de Khaya anthotheca}

Les résultats de l'étude ont montré que la densité de régénération varie en fonction des classes de diamètre et également en fonction des concessions (milieu). Les densités des plantules de $K$. anthotheca trouvées dans ce travail, sont similaires à celles obtenues par Kasaï (2007) en RDC sur la même espèce. Il faut souligner cependant que la distribution des semis et donc des plantules est conditionnée par le mode de dissémination de l'espèce. Que cela soit en forêt primaire moins perturbée ou en forêt secondaire, soumises aux pressions humaines diverses, l'action du vent sur les diaspores de cette espèce est fortement réduite par le couvert végétal. Les semis tombent sous la couronne végétale et il y a forte densité principalement autour et en périphérie de la limite de la couronne. Par conséquent, les diaspores sont regroupées et ne sont pas réparties uniformément sur le sol. Cependant, il faut remarquer que malgré l'action fortement réduite du vent dans le sous-bois par la couronne végétale, l'anémochorie n'est pas pour autant nulle. Outre la couronne, les branches du pied porteur ou celles des arbres et arbustes environnants, constituent aussi un obstacle pour la chute des diaspores (Kasaï, 2007). La mortalité des plantules est aussi provoquée par la prédation. Les insectes s'attaquent aux feuilles des plantules et les détruisent. L'auteur a poursuivi en soulignant que l'environnement exerce sur la régénération deux effets majeurs : d'une part la dispersion et la prédation des graines par les animaux. D'autre part, le jaunissement des feuilles et le recouvrement du bord des limbes sont des signes qui ne trompent pas sur l'attaque de différents agents pathogènes. Enfin, aucune direction ne semble être préférentielle pour les plantules de $K$. anthotheca tant sur le PEA 192 que sur le PEA 188. Une étude de ce genre effectuée au Gabon (Doucet, 2003) a montré que la densité la plus élevée des plantules de Baillonella toxispermia (Moabi), était trouvée au Nord-Est (40 \%) tandis que celle des plantules de 
Entandrophragma congolense, (Tiama noir), était obtenue au Nord et à l'Ouest $(40 \%)$. D'après Gobert (2002), il n'existe pas de direction préférentielle d'installation du semis en forêt dense tropicale humide où le vent ne souffle pas dans une direction bien définie.

\section{Conclusion}

Les résultats obtenus dans le cadre de cette étude montrent une faible représentativité des individus de $K$. anthotheca dans les deux concessions avec une forte décroissance de l'Ouest vers l'Est du massif forestier. Au niveau des deux concessions forestières, la distribution des effectifs par classe de diamètre a permis d'obtenir deux courbes exponentielles décroissantes des petits diamètres vers les gros diamètres. Ce qui suppose un fort potentiel de régénération naturelle de cette espèce en l'absence des perturbations liées aux pressions anthropiques. Comme pour la structure diamétrique, les surfaces terrières présentent des valeurs plus importantes sur le PEA 188 (oscillant autour de 1 pour les petits diamètres) tandis que celles-ci sont réduites sur le PEA 192. Ceci est en lien avec la rareté des individus de cette espèce dans cette concession du fait de la pression des usages humains. Ces différentes pressions sont notamment à l'origine de la régression du nombre des individus de gros diamètre dans les deux concessions forestières avec de fortes baisses observées sur le PEA 192. L'étude a permis aussi de confirmer qu'il n'existe pas de direction préférentielle de régénération pour le $K$. anthotheca. De tout ce qui précède, il est possible d'affirmer que les peuplements de $K$. anthotheca sont en constante régression dans le massif forestier $\mathrm{du}$ Sud-ouest avec un gradient sans cesse croissant de l'Ouest (PEA 188) vers l'Est (PEA 192) plus exposé aux pressions anthropiques venant de la capitale Bangui. L'étude a permis de constater à nouveau que l'interdiction de l'exploitation forestière industrielle n'endigue pas le risque de disparition des essences forestières rares du Sud-Ouest du pays du fait de la persistance des menaces liées aux autres usages par les populations. Elle a permis de mieux connaître les caractéristiques des peuplements naturels des espèces qui sont en danger d'extinction. L'étude met enfin en évidence la nécessité d'initier des travaux de recherche sur la régénération artificielle de $K$. anthotheca dans les conditions pédoclimatiques du Sud-Ouest de la RCA. Cette étude pourra aboutir à la proposition d'intégration systématique de cette espèce dans les programmes de restauration forestière par l'agroforesterie et sa valorisation durable comme produits forestiers non ligneux au profit des populations dépendantes des ressources naturelles.

\section{CONFLIT D'INTERETS}

Aucun conflit d'intérêts n'est signalé par les auteurs.

\section{CONTRIBUTIONS DES AUTEURS}

Protocole de collecte des données : BD, JJL et GIT; collecte des données : BD ; analyse des données et rédaction du manuscrit : $\mathrm{BD}$, GIT, et JJL ; relecture du manuscrit : JJL et ODY.

\section{REMERCIEMENTS}

Les auteurs remercient tous ceux qui ont contribué à la réalisation de ce travail. Ces remerciements vont tout d'abord aux responsables du Ministère des Eaux, Forêts, Chasse et Pêche (MEFCP) qui ont accordé leur autorisation pour la conduite de ces travaux de recherche dans les concessions forestières de CentraBois et Timberland ainsi qu'à l'Agence de gestion Durable des Ressources Forestières (AGDRF) qui a mis à leur disposition les données des inventaires forestiers. Enfin, les remerciements vont particulièrement aux lecteurs anonymes, qui ont accordé de leur temps si précieux pour relire ce manuscrit.

\section{REFERENCES}

Ahissou MV, Balagueman OR, Biaou SSH, Natta AK, Dan BSC. 2017. Caractérisation structurale des populations de Borassus aethiopum Mart., dans la commune de Savè au Bénin. Annales UP, Série Sciences Naturelles et Agronomie, 7 (1) : 47-53.

Bamba I, Barima Y, Bogaert J. 2010. Influence de la densité de la population sur la structure spatiale d'un paysage forestier dans le bassin du Congo en R. D. Congo. Tropical Conservation Science, 3(1):3144. DOI: 10.1177/194008291000300104. 
Baye-Niwah C, Todou G, Souré K, Abdoulaye A, Bay S et Atem E, 2020. Diversité et usages des plantes ligneuses des agrosystèmes périphériques de la ville de Maroua (Extrême-Nord, Cameroun). International Journal of Biological and Chemical Sciences, 14(3): 966-982. DOI: https://doi.org/10.4314/ijbcs.v14i3.25.

Béina D. 2011. Diversité floristique de la forêt dense semi-décidue de Mbaïki, République Centrafricaine : étude expérimentale de l'impact de deux types d'intervention sylvicole, Thèse de doctorat Unique, Université de Picardie Jules Verne, p. 226.

Bouka Dipelet UG, Doumenge C, Loumeto JJ, Florence J, Gonmadje C, McKey D. 2019. Des confusions entre espèces préjudiciables à la gestion durable des essences forestières : l'exemple des acajous d'Afrique (Khaya, Meliaceae). Bois et Forêts des Tropiques, 339 : 17-32. DOI:

https://doi.org/10.19182/bft2019.339.a31 714.

Deguene B, Touckia IG, Semboli O, Loumeto JJ. 2018. Analyse ethnobotanique des essences rares des concessions forestières du Sud-Ouest de la RCA. Rev. RAMRES, 6 (2): 63-70.

Dossa BAK, Sourou B, Ouinsavi C. 2020. Germination des Graines et Croissance en Pépinière et en Champ des Plantules de Detarium senegalense au Bénin. European Scientific Journal, 16(12). DOI: 10.19044/esj.2020.v16n12p38.

Doucet JL. 2003. L'alliance délicate de la gestion forestière et de la biodiversité dans les forêts du centre de Gabon. Thèse de doctorat, Faculté des sciences agronomiques de Gembloux, p. 323.

Gaoue O, Ticktin T. 2007. Patterns of harvesting foliage and bark from the multipurpose tree Khaya senegalensis in Benin: variation across ecological regions and its impacts on population structure. Biological Conservation, 137: 424-436. DOI: $\quad$ https://doi.org/10.1111/j.13652664.2007.01381.x

Gbesso F, Yedomonhan H, Tente B, Akoegninou A. 2014. Distribution géographique des populations de rôniers
(Borassus aethiopum, Arecaceae) et caractérisation phytoécologique de leurs habitats dans la zone soudano-guinéenne $\mathrm{du}$ Benin. Journal of Application Biosciences, 74 : 6099-6111. DOI : http://dx.doi.org/10.4314/jab.v74i1.14.

Gobert A. 2002. Etude de la régénération naturelle de quelques essences commerciales au Gabon. Mémoire inédit, Faculté universitaire des sciences agronomiques de Gembloux, p. 75.

Gouwakinnou GN, Kindomihou V, Assogbadjo AE, Sinsin B. 2009. Population structure and abundance of Sclerocarya birrea (A Rich.) Hochst. subsp. birrea in two contrasting land-use systems in Benin. International Journal of Biodiversity and Conservation, 1 (6): 194201. DOI: 10.5897/IJBC.

Hawthorne W, Jongkind C. 2006. Woody plants of western African forests: a guide to the forest trees, shrubs and lianes from Senegal to Ghana. Kew Publishing (ed). Royal Botanic Gardens: Kew; 909-923.

Houessou GL, Lougbegnon OT, Gbesso GHF, Anagonou SEL, Sinsin B. 2012. Ethnobotanical study of the African star apple (Chrysophyllum albidum G. Don) in the Southern Benin (West Africa). Journal of Ethnobiology and Ethnomedicine, 40(8): 1-10. DOI: 10.1186/1746-4269-8-40.

Kasaï Kikango J. 2007. Observations préliminaires sur la régénération de Khaya anthotheca (Wew.) C.DC. « Acajou d'Afrique » dans la Réserve forestière de Yoko, bloc sud, Ubundu, RDC. Mémoire en vue de l'obtention du diplôme de Licencié en Biologie, Université de Kissangani, RDC, p. 11.

Kourouma K, Dissous FE, Ganglo, JC. 2013. Caractérisation écologique et structurale des parcs à Néré (Parkia biglobosa (Jacq.) R. Br. Ex.G.Don) du département de Donga au Nord-Ouest du Bénin. International Journal of Biological and Chemical Sciences, 7(2): 726-738. DOI: 10.4314/ijbcs.v7i2.27.

Makana JM, Thomas SC. 2004. Dispersal limits natural recruitment of African mahoganies. Oikos, 106 (1): 67-72. DOI: 10.1111/j.0030-1299.2004.13084.x. 
Maroyi A. 2008. Khaya anthotheca (Welw.) C.DC. In Plant Ressources of Tropical Africa, Louppe D, Oteng-Amoako AA, Brink M (eds). PROTA Foundation: Wageningen, Netherlands / Backhuys Publishers: Leiden, Netherlands/ CTA: Wageningen, Netherlands; 7-21.

MEFCP. 2019. Le Plan d'Aménagement du Permis d'Exploitation et d'Aménagement (PEA) de l'entreprise forestière Timberland. Agence de Gestion Durable des Ressources Forestières (AGDRF) et le Projet de Développement de la Région du Sud-Ouest (PDRSO): Groupement CIRAD-Forêt et FRM ; 63-81.

MEFCP. 2018. Le Plan d'Aménagement du PEA de l'entreprise forestière Centrabois. AGDRF et le PDRSO : Groupement CIRAD-Forêt et FRM ; 94-109.

Neuenschwander P, Sinsin B, Goergen G. 2011. Protection de la Nature en Afrique de l'Ouest: Une Liste Rouge pour le Bénin. Nature Conservation in West Africa: Red List for Benin. International Institute of Tropical Agriculture, Ibadan, Nigeria, p. 36.

Ofori DA, Opuni-Frimpong E, Cobbinah JR. 2007. Provenance variation in Khaya species for growth and resistance to shoot borer Hypsipyla robusta. Forest Ecology and Management, 242: 438-443. DOI: 10.1016/j.foreco.2007.01.090.

Orékan VA. 2007. Implémentation du modèle local CLUE aux transformations spatiales dans le Centre Bénin aux moyens de données socio-économiques et de télédétection. Thèse de doctorat, Université de Bonn, Allemagne, p. 204.

Réjou-Méchain M, Pélissier R, Gourlet-Fleury S, Couteron P, Nasi R, Thompson JD. 2008. Regional variation in tropical forest tree species composition in the Central African Republic: an assessment based on inventories by forest companies. Journal of Tropical Ecology, 24: 663-674. DOI: https://doi.org/10.1017/S0266467408005 506.

Sinsin B, Eyog Matig O, Assogbadjo AE, Sinadouwirou T. 2004. Dendrometric characteristics as indicators of pressure of Afzelia africana Sm. Dynamic changes in trees found in different climatic zones of Benin. Biodiversity Conservation, 13: 1555-1570.

DOI: https://doi.org/10.1023/B:BIOC.0000021 328.56517.46.

Sourou BN, Ouinsavi C, Sokpon N. 2016. Ecological Structure and Fruit Production of Blood Plum (Haematostaphis barteri Hook F) Subpopulations in Benin. International Journal of Plant and Soil Science, 9 (2): 1-12. DOI: 10.5897/IJBC.

Touckia GI, Kosh-komba E, Yongo OD. 2014. Plantes alimentaires d'intérêt médicinal utilisées par les Pygmées de la commune de Pissa, République Centrafricaine. International Journal of Biological and Chemical Sciences, 8: 517-527. DOI : http://dx.doi.org/10.4314/ijbcs.v8i2.11.

Traoré L, Ouédraogo I, Ouédraogo A, Thiombiano A. 2011. Perceptions, usages et vulnérabilité des ressources végétales ligneuses dans le Sud-Ouest du Burkina Faso. International Journal of Biological and Chemical Sciences, 5(1): 258-278. DOI: 10.4314/ijbcs.v5i1.68103.

Traoré L, Sambaré O, Savadogo S, Ouedraogo A, et Thiombiano A. 2020. Effets combinés des facteurs anthropiques et climatiques sur l'état des populations de trois espèces ligneuses vulnérables. International Journal of Biological and Chemical Sciences, 14(5): 1763-1785. DOI: https://doi.org/10.4314/ijbcs.v14i5.21. 